\title{
Infrastructuring Transdisciplinary Problem Solving in Translational Research
}

\author{
Santosh BASAPUR ${ }^{\mathrm{a}, \mathrm{b}, 1}$, Keiichi SATO ${ }^{\mathrm{b}, \mathrm{c}}$, \\ Raj C. SHAH ${ }^{\mathrm{a}}$ and Sherry ROBISON ${ }^{\mathrm{d}}$ \\ ${ }^{\text {a }}$ RADC, Rush University Medical Center, USA \\ ${ }^{\mathrm{b}}$ Illinois Institute of Technology, USA \\ ${ }^{c}$ UiT The Arctic University of Norway, Norway \\ dITM, University of Chicago, USA
}

\begin{abstract}
Translational Research in the health sciences endeavors to bring biomedical discoveries into clinical applications that improve human health. This work is complex and long-term with a substantial risk of failure. A key step of clinical trials is needed to evaluate the effects of those interventions on human biomedical or behavioral outcomes. Timely recruitment of human subjects and meeting recruitment milestones is recognized as one of the most significant contributors to delays and failures. Quality and Design approaches have been tried to address the problem but the scope has been limited. We proposed to determine how Quality and Design may lead to complementary solutions for these barriers of Translational Research. The first ten studios using this approach are presented here. Three themes emerged: (1) problems were investigated similarly but there was a difference in insights, (2) quality process-based solutions tended to be specific to the issue discussed whereas the design process often yielded solutions broader or even tangential, and (3) quality solutions demonstrated more immediacy while design solutions showed more systemic ideas. In conclusion, the paper demonstrates how Design and Quality in a transdisciplinary studio may lead to solutions with different characteristics for clinical trials and advance translational science.
\end{abstract}

Keywords. Transdisciplinary methods, design approach, quality approach, clinical trials, translation, translational science, human centered design, systems design

\section{Introduction}

Translational Research, specifically clinical trials, endeavors to bring biomedical discoveries into clinical applications that improve human health [1] [6]. Translation is a complex, long-term, multi-stakeholder process. It is a high-cost quest with substantial risk of failure. Clinical trials involving human participants are a key late stage step in the translation process. Barriers in initiating and completing clinical trials have been well documented [20]. Research shows how significant delays (years or decades) occur in stages of clinical research study start-up, protocol initiation and study implementation, before a successful implementation results in health benefits for patients, communities and/or providers of care [4]. Timely recruitment and meeting milestones is recognized as one of the most significant contributors to clinical trial delays and completion failures. Inefficiencies have traditionally been addressed using quality techniques [7].

\footnotetext{
${ }^{1}$ Corresponding Author, Mail: santosh_basapur@rush.edu.
} 
National Center for the Advancement of Translational Sciences (NCATS) at the NIH (including their Clinical and Translational Sciences Award (CTSA) mechanism) is dedicated to advancement of translational sciences. One national CTSA Central program is the Trial Innovation Network [21]. NCATS has created CTSA Program hubs and partners with them to develop and implement innovative, collaborative solutions intended to transform clinical and translation research including through the Trial Innovation Network [22]. The Institute for Translational Medicine (ITM) in Chicago, Illinois, is one of the CTSA hubs funded by NCATS. ITM is composed of 6 Chicagoland health institutes and medical colleges. ITM is a cross-institutional, transdisciplinary endeavor that develops workforce, demonstrates solutions for patient and community engagement, and disseminates holistic approaches for advancing translation as a science. ITM also focusses on equity across underserved populations, process innovation for quality and efficiency of multisite trials through integration of informatics [15] [16].

Translation by nature needs to be inter-disciplinary to deliver interventions and care from the basic sciences research [23]. The interdisciplinary training of future translational workforce is much needed. not a want but a need. The work conducted in this field is often from multiple dynamic and evolving systems including health, clinical care delivery, quality of care, and medical and related sciences. Translation teams could certainly collaborate with others of different disciplines, but there are barriers. An alternative is to teach problem solving skills inspired by multi-disciplinary approaches to reduce the burden of studies and research projects and enable collaboration [24]. Inefficiencies have traditionally been addressed using quality techniques [7].

Trial recruitment and retention innovation is being developed using structured approaches to problem solving [17]. Popular approaches in health care systems are that of quality science. Recentlysome adoption of design science, including human centered design [10], [11] has taken place with the popularity of design thinking based innovation. As part of the ITM's Trial Recruitment Innovation Office (TRIO), transdisciplinary studios were conceptualized. To innovate and address the legacy barriers in the clinical trials, the researchers implemented approaches based in design and quality methods. This paper describes the studios and the design and quality science methods used in the studios and the results found. Research team conducted approximately monthly in-person studios of 90 minutes from December 12, 2017 to September 11, 2019. The studios continue to be held to date. This paper focusses on the the first 10 studios with Design and Quality approaches done simultaneously and reports on the outcomes observed.

\section{Research Objectives}

The goal of this research is to infrastructure resiliency in translational research teams. Main objective is to determine if design methods and quality methods can accelerate clinical research by solving recruitment and retention issues of participants. Furthermore, this research aspired to determine how the various tools of design and quality approaches help the study teams in different situations and contexts.

\section{Literature Review}

Translation is the "process of turning observations in the laboratory, clinic and community into interventions that improve the health of individuals and the public."[1] 
Translational research is defined by NCATS as the endeavor to traverse a step of the translational process for a specific target or disease. Translational science seeks to understand the scientific and operational principles underlying each step of the translational process [1].

This process of converting medical science advances into applied bedside interventions for patients has two main road blocks in the transfer of research knowledge into practice. The first is barriers that prevent laboratory advances from being converted into new medical products and the second is inability to enable adoption of proven improvements in treatment in medical practice for instance new drug combination [5] [6]. Barriers to patient recruitment especially diverse participants from ethnic or racial minorities consist of family composition, literacy, and severity of medical diagnosis [7].

The Principal Investigators (PIs) and the study team for clinical trials also face barriers of three kinds [8] [9]. Firstly, PIs have to work with many clinicians who are either not aware of active trials or do not refer their patients for participation in trials. Secondly, substantial costs are necessary to enroll and retain diverse populations. For minorities, extra time is needed to gain individual and community acceptance [7]. Finally, administrative burdens and regulatory requirements present unprecedented challenges, even for experienced trialists [8] [9].

Substantial effort has gone into addressing the barriers over the years. NCATS has funded many such efforts. Researchers have successfully applied and demonstrated that better coordination, timeliness, efficiency and value of clinical and translational research can be achieved by applying the Lean and Six Sigma principles [4]. In recent years [10] [15] it has been successfully argued that Human Centered Design (HCD) has been found to offer specific methods that can readily operationalize implementation strategies to improve the translation of health innovations into practice. Using HCD to identify and execute strategies provides a set of tools for researchers to develop and test health interventions. Implementation scientists refer to this as application of design thinking to health care research [16] [19].

\subsection{Quality Approach (QA)}

Quality Approach focuses on understanding a situation by breaking the problem into parts. Deductive reasoning is used to then intervene to eliminate process failure(s) [4].

Many healthcare service providers and some research organizations have been adopting quality science-based approaches (QA) in order to improve their organizational performances both in practice and research [4] [15]. Although QA has been proven to be very effective for solving problems and enhancing performances, there are still problems that require approaches in addition to QA. QA methodology has been developed and widely applied in many industries like auto manufacturing, electronic goods and transportation. Previously, quality improvement methodologies have been adopted in approaching system problems associated with health care delivery and (to some extent) research processes. For instance, root cause analysis and failure mode effectiveness analysis have been imported in the health care sector from the manufacturing sector in order to achieve reliable and consistent outcomes [4] [7].

This research has adapted the traditional DMAIC - Define, Map the process, Analyze the causes, Implement and Control approach of Total Quality Management for solving issues and barriers faced by a study team and PI. DMAIC is much more closer to Human Centered Design process so it was easier to compare and contrast as seen in Figure 2 . 


\subsection{Design Approach (DA)}

Design Approach focuses on envisioning a solution to best meet the needs of the endusers and other stakeholders. It takes a holistic, abductive approach to problem solving rather than a focus on perfection and avoiding failure at all costs. Early prototyping and accepting failure to build better processes are key learning tools and seminal features of design science thinking [18].

Design Approaches (DA) also have been introduced at different levels in order to address human perspectives from different stakeholders in healthcare systems [18]. Although the term and approaches of "design thinking" have been widely adopted [15] [16] and generated some success stories in many areas in order to enhance innovation capability in organizations, yet no universal understanding has been formed or accepted for translational research. This research constructs its own DA based on the methods proven to be effective in practice [18] [19].

Design methodologies were first described approximately the same time as quality improvement methodologies; however, while their implementation has started to accelerate in other sectors, they have only very recently been introduced in the health care arena. This research has adapted the traditional HCD process into - Sense Intent, Know Context, Know People, Frame Insights, Explore Concepts and Frame Solutions to a five step process to solve the translational research problems. Figure 2 below shows the two methods side by side as well as elaborates on some of the tools used in each step.

However, no studies have examined whether design is comparable to quality methodology in producing impactful changes in the process of translational research.

\section{Method}

Collaborative studios with multiple stakeholders were created and sessions conducted to test this approach. The researchers focused on using the concept of collaborative studios to trouble shoot the issues of translational research studies including start up issues as well as recruitment and retention issues. Researchers adapted quality approach and design approach as the framework for problem solving translation research issues. In a studio, principal investigators of translational projects presented their problematic recruitment/clinical trial scenario to TRIO Studio. The studio participants then worked as a group towards solving the issues and suggesting systematic solutions using both Design and Quality processes. The methods have been adapted to fit $90 \mathrm{~min}$ sessions format. The studios happened on a monthly basis.

\subsection{TRIO Studio process}

Preparation for a TRIO studio included setting up of facilities, ensuring the template is shared with the problem presenter and iterating with the presenter on importance of concluding their presentation with a clear statement of problem the group needs to focus on. Also, the facilitator studied the problem beforehand and created prompts and other facilitation materials for the Studio.

Studio participants (SMEs) attended from across six Chicagoland institutions with significant subject matter expertise in Translational Research. SMEs included research regulatory science, informatics and data analytics, community relations and/or research 
operations. Experts interested in the topic and willing to provide insight and expertise to the problem attended the studios as per their schedule convenience. All studios were facilitated by an experienced researcher. Facilitator are responsible for the adherence to methods. The TRIO team ensured that the discussion was active and all SMEs were contributing.

Each studio started with the TRIO Manager welcoming the multi-institutional attendees. A given studio could be as small as 5-6 people or as large as 40 people. The facilitator then introduced the two approaches of QA and DA. The presenter of the day described their project, explained the current state and the problem(s) in executing the particular recruitment and retention strategy.

The TRIO Design Studio participants were then randomly assigned in each session to use design methodologies or quality methodologies for problem-solving. Once assigned, teams worked in parallel to develop solutions during approximately an hour of work in their assigned methodology to address barriers in steps associated with accelerating clinical research initiation and recruitment in the project presented by investigator(s).

The Facilitator enabled the studio attendees to navigate through the session with the methods chosen to address the presenter's problem(s). TRIO staff from the ITM institutions helped with set up and documentation of the studio. The TRIO staff also assisted with moderation as needed - drawing on whiteboards, taking notes, tendering surveys etc. With permission of the attendees, the sessions were audio and videorecorded for program fidelity and qualitative analysis for generating reports.

The actual studio proceeds as listed below in Figure 1:

\begin{tabular}{|c|c|c|}
\hline \multicolumn{3}{|c|}{ Welcome and Introduction of TRIO Studio TRIO Manager } \\
\hline \multirow{9}{*}{$\begin{array}{l}\text { Method(s) } \\
\text { introduction } \\
\text { TRIO Facilitator }\end{array}$} & \multicolumn{2}{|c|}{ Ground Rules of the Studio are described by Moderator } \\
\hline & \multicolumn{2}{|c|}{ Presenter presents the case and the problem needing solutions } \\
\hline & Design Science Approach (DSA) & Quality Science Approach \\
\hline & Sense Intent - Mind Map & \multirow{2}{*}{$\begin{array}{l}\text { Define Problem and Describe details - Current state, } \\
\text { future state, and scale of problems }\end{array}$} \\
\hline & Know Context - Stakeholder Map & \\
\hline & Generate Insights - How Might We Post its & Map the Process - Identify leaks using leaky pipe model \\
\hline & Ideate Solutions - SCAMPER Tool & Analyze the causes of effects observed \\
\hline & \multirow{2}{*}{$\begin{array}{l}\text { Prioritization of solutions for study team to try } \\
\text { systematically }\end{array}$} & Ideate Solutions - SCAMPER Tool \\
\hline & & $\begin{array}{l}\text { Prioritization of solutions for study team to try } \\
\text { systematically }\end{array}$ \\
\hline
\end{tabular}

Case Presentation Principal Investigator of the Project or the study team
$\begin{aligned} & \text { Studio } \\ & \text { Group splits into } 2 \text { and works on the problem with facilitation as necessary } \\ & \text { Group Presentation and Share-0ut } \\ & \text { Discussion with presenter on the solutions emerged }\end{aligned}$

Announcements and Wrap up

Post-Studio Follow up 30 days, 90 days and 1 year

Figure 1. TRIO Studio Process.

Participants received handouts explaining the methods of Design and Quality. The handout is shown in Figure 2 below. 


\section{Design Approach}

Sense Intent

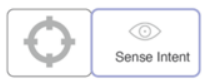

01 Method: Mind mapping

\section{Know Context}

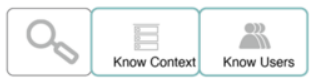

02 Method: Stakeholder mapping

Generate Insights

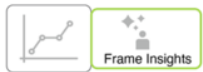

03 Method: Create How Might Ne, statements

\section{Idea \& Create Solutions}

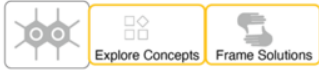

04 Method: Idea based on insights and build solutions as a group

\section{Explore to understand} study "Intent"

Know the people with roles in the study

Discuss "all stakeholders"

Identify the problems and reframe them into opportunities

Ideate as a group and build on ideas to create solutions. Prioritize the solutions.

\section{Quality Approach}

\section{Define Problem}

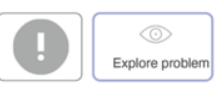

01 Method: Problem mapping template

\section{Map the Process}

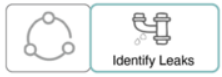

02 Method: Map the process of recruitment and retention

\section{Analyze Causes}

\begin{tabular}{|c|c|}
\hline 54 & Analyze Cause \& Effects \\
\hline
\end{tabular}

03 Method: Fish and Bone Diagram

\section{Idea \& Create Solutions}

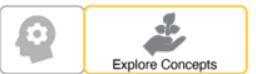

04 Method: Ideate solutions, pilot and manage change
Explore the problem current state and goal state

Create a leaky pipe model of the study team process for recruitment and retention

Get deep understanding of causes behind problems seen.

Create solutions to causes identified. Prioritize the solutions as a group.

Figure 2. Illustrated DA and QA handouts for studio participants. 
Post-Studio work included survey collection for feedback about solutions and session itself. After each studio,a report was created with in 2 weeks, describing the studio and the solutions generated during the session. Reports were shared with the presenter as well as the attendees. The reports also were hosted on a public wiki page for easy access by anyone interested. Also, the TRIO Manager followed up with the study team at 30 days, 90 days and 1 year timepoints. That feedback from those timepoints also was rolled into the reports with an addendum. For this paper, 10 initial studio reports were analyzed. Problem characteritics, participant attendance as well as qualitative data from the session solutions were analyzed using constant comparative coding.

\section{Results}

Analysis of 10 TRIO Studios with participation varying from 5-24 SMEs (median $=9$ is presented here. At each studio, participants were assigned to Design or Quality team and they accordingly used design or quality methods to address issues faced by a clinical research team. Figure 3 shows the number of participants per studio and Figure 4 shows the nature of problems addressed.

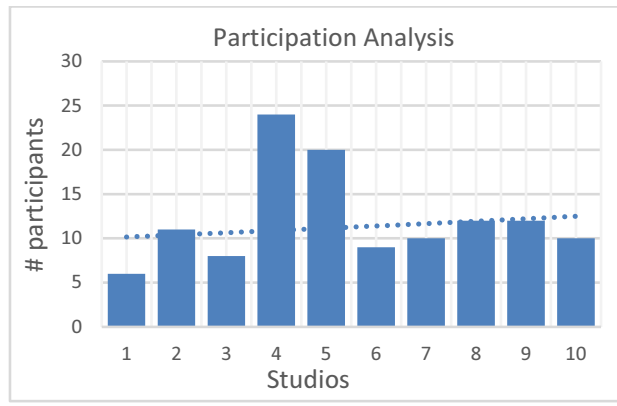

Figure 3. Participation in Studios.

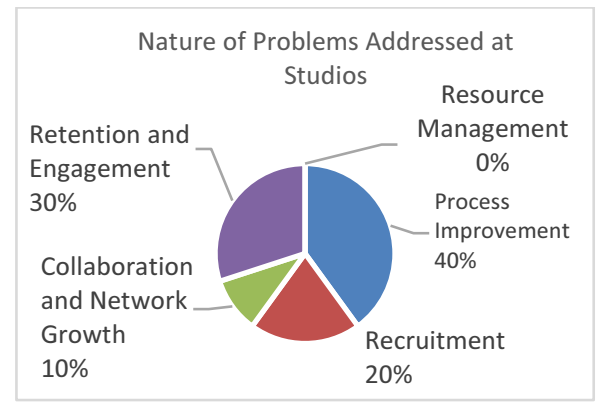

Figure 4. Nature of problems addressed.

The qualitative data of the 10 studio reports was analyzed. The analysis showed that not only did the nature of problems affect the kind of solutions generated by the group but also the method used. In 4 of out 10 studios QA actually produced a greater number of solutions than DA. However, when the solution set was analyzed, the solutions from each approach were not all of the same granularity. In QA the ideas/solutions appeared to be more targeted to problem due to the nature of the process of quality improvement. In DA the ideas/solutions, even though many times less than QA, were broader and even tangential to the exact problem defined by the presenter. In DA, due to explicit step of reframing, the attendees paid more attention to context and pivoted into things that sometimes the study team presenter did not even define at the outset.

For issues related to clinical research initiation and recruitment, quality and design solutions offered complementary approaches. Three presenters (out of 10) selected and retained the use of design solutions over a 12 month follow-up period [16]. Analysis of reports showed nuances in how participants address problem when given design versus quality methods and tools. Three main themes emerged: 


\subsection{Theme 1: Nature of process guides focus of causality analyses}

Problems were investigated in both DA and QA by using relevant tools provided by the facilitator. There was significant difference in insights developed by the two approaches. Due to nature of the processes, DA insights tended to be about stakeholders and QA insights tended to be about process failure points. The tools used directly influenced the group's focus and thinking.

For instance in one particular studio the problem was that of inter-institute collaboration and the idea floated by QA was that of Institutional Review Board (IRB) procedure optimization and DA proposed camping out in the cafeteria and engaging people when they come to café for their breaks.

\subsection{Theme 2: Approaches shaped the degrees of freedom of the ideating team}

Interestingly, QA-based solutions tended to be specific to issue discovered and discussed by the group. Alternatively, in DA, design process often took the discussion to stakeholders who may or may not be directly involved in the given problem. Often this yielded solutions that were broader or at times tangential to the issues discussed.

For instance in a studio on food and diet study for longer life and well being of people, QA team delved into data needs and so their solution was an informatics approach to recruitment in a minority population. On the other hand, DA team, the data need was discussed similar to QA team but the solution was quite different. The DA team explored the stakeholder map and converted the clinician who knows their patients well as the recruitment ambassador. From the study team point of view this was a win-win because they got two very different solutions that they could pilot. The difference between QA based solution and DA based solution was stark.

\subsection{Theme 3: Depth and breadth of solutions definitely varied by approach used}

Quality solutions demonstrated more immediately implementable solutions while design solutions showed more systemic ideas. For instance in a studio on "End of Life Care" the QA group focused on review of training given to nurses as a solution and how to optimize it. Meanwhile the DA team reframed the problem from local to national level. They decided that it is an urgent topic and needs NCATS attention and resources. So a national consortium of collaborators with NCATS funding was proposed as a better solution. Figure 5 below shows the variations.

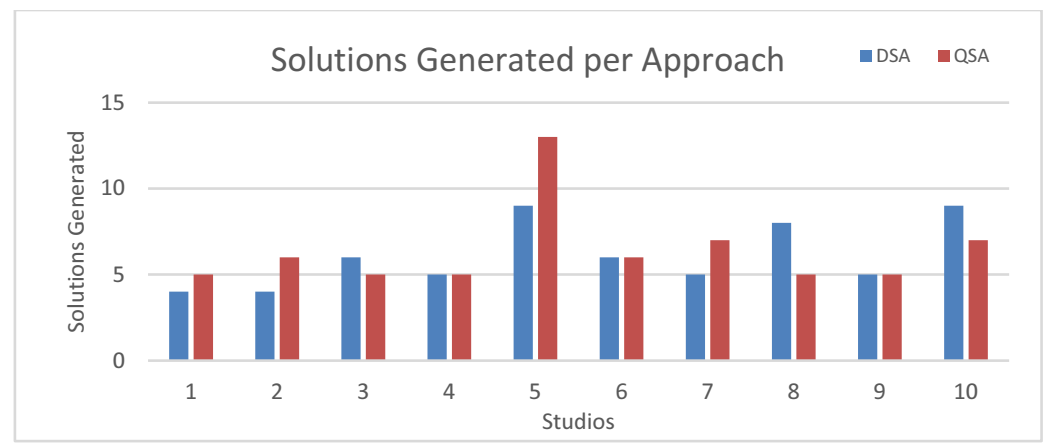

Figure 5. Solutions created per studio (using both DA and QA) 


\section{Discussion}

Through this effort, the techniques of problem solving are being democratized and made familiar to the clinical research study teams. The study teams will directly impact translational science growth as well as build resiliency to challenges of their field. Structured approach helps teams to learn the skill and use it in their day to day work. Also as approaches become repeatable and reliable, the teams become more adaptable to changes in context. For instance, most work went virtual during COVID 19 and translational research had to rapidly adapt itself.

\subsection{Advancing the Translational Research}

Clinical research initiation, recruitment and retention were addressed holistically by quality and design solutions. These approaches offered complementary ideas and solutions revealing the need for multi-pronged approach. The presenters who did chose design solutions over quality solutions and used them over a 12 month follow-up period. This shows the impact of creative and collaborative solutioning on translational research.

Furthermore, this approach of solving translational science issues with both DA and QA is an example of how to compare and contrast methods from different disciplines [11] [12]. Usually quality and design teams are pitted against each other. This research shows that the teams and their approaches are more complementary than mutually exclusive. When targetted investigation of causality of effect observed in an established process when QA might work better.

\subsection{Implications for Transdisciplinary Engineering}

Integral approach discovered for problem solving in translational research can inspire approach to problem solving in other domains such as industry 4.0 and cyber physical systems engineering. When human centered analysis, identification of problems, and better fit between people and technology solutions are needed then Design Approach might better fit the needs of research teams [18] [19]. TE researchers can utilize these tools and adapt the approach and experiement in their respective domains.

\subsection{Strengths and Weaknesses}

Strengths of this research is the scale at which the TRIO studios are being conducted. The research is now running in its $3^{\text {rd }}$ year so diversity of case studies, diversity of SMEs at the table in consistent manner and the execution of studios has been a revelation of how well it has worked out. On the other hand, the sheer size of the operations itself can be a deterrent for someone else to try to replicate this approach for their domain. There can be lot of logistical issues and space availability issues for such a large scale approach.

\subsection{Future Work}

Dissemination and implementation of studio facilitation tools and knowledge bases is next step. Further work is needed to understand which types of problems are best amenable to a particular methodology. Researchers are exploring collaborations with other TE researchers and apply this method in domains other than translational science. 


\section{Acknowledgement}

This project was supported by the National Center for Advancing Translational Sciences (NCATS) of the National Institutes of Health (NIH) through Grant Number 5UL1TR002389-02 that funds the Institute for Translational Medicine (ITM). The content is solely the responsibility of the authors and does not necessarily represent the official views of the NIH. TRIO leadership is indebted to the presenters, Subject Matter Experts and other attendees as well as our TRIO Staff and numerous students.

\section{References}

[1] C.P. Austin. Translating translation. Nature Reviews: Drug Discovery. July 2018; 17: pp. 455-456.

[2] A. Dong, D. Lovallo, and R. Mounarath. The effect of abductive reasoning on concept selection decisions. Design studies, 2015, Vol. 37, pp. 37-58.

[3] D.L. Dean, J.M. Hender, T.L. Rodgers, and E. Santanen. Identifying good ideas: constructs and scales for idea evaluation, Journal of Association for Information Systems, 2006, Vol. 7, Issue 10, pp. 646-699.

[4] S.A. Schweikhart and A.E. Dembe. The applicability of Lean and Six Sigma techniques to clinical and translational research. Journal of Investigative Medicine, 2009, Vol. 57, Issue 7, pp. 748-755.

[5] D. Butler, TR: crossing the valley of death. Nature News, 2008, 453(7197), pp. 840-842.

[6] S.H. Woolf, The Meaning of TR and Why It Matters. JAMA, 2008, 299 (2), pp. 211-213.

[7] S.J. Haley, L.E. Southwick, N.S. Parikh, J. Rivera, D. Farrar-Edwards, B. Boden-Albala, Barriers and Strategies for Recruitment of Racial and Ethnic Minorities: Perspectives from Neurological Clinical Research Coordinators. J Racial Ethn Health Disparities. 2017, Vol. 4(6), pp.1225-1236.

[8] J.L. Probstfield, R.L. Frye, Strategies for Recruitment and Retention of Participants in Clinical Trials. JAMA. 2011, 306(16):1798-1799. doi:10.1001/jama.2011.1544.

[9] R.B. Gul and P.A. Ali, Clinical trials: the challenge of recruitment and retention of participants. Journal of Clinical Nursing, 2010, Vol. 19, pp. 227-233.

[10] E. Chen, G. Neta, M.C Roberts, Complementary approaches to problem solving in healthcare and public health: implementation science and HCD, Transl. Behavioral Medicine, 2021, Vol. 11, pp. 1115-1121.

[11] A. Doshi and C. Clay, Rethink space: (Re)designing a workspace using human-centered design to support flexibility, collaboration, and engagement among clinical and translational research support services." Journal of Clinical and Translational Science, 2017, Vol. 1, pp. 160 - 166.

[12] Schmidt, S., Shay, L. A., Saygin, C., Schulz, K., Clark, R. A., \& Shireman, P. K. Improving pilot project application and review processes: A novel application of lean six sigma in translational science. Journal of clinical and translational science, 2018, Vol. 2(3), pp. 135-138.

[13] P.S. Gillam, H.B. Nembhard and D.A. Munoz, The role of quality improvement methods in translational research. Proc. of the 2014 Industrial and Systems Engineering Research Conf., 2014, pp. 4023-31.

[14] J.M. Glasgow, J.R. Scott-Caziewell and P.J. Kaboli, Guiding inpatient quality improvement: a systematic review of Lean and Six Sigma. The Joint Commission Journal on Quality and Patient Safety, 2010, Vol. 36(12), 533-540.

[15] A.R. Dopp, K.E. Parisi, S.A. Munson, A. and R. Lyon, A glossary of user-centered design strategies for implementation experts. Transl Behav Med. , 2019, Vol. 25;9(6), pp. 1057-1064.

[16] R. Yajima, Catalyzing scientific innovation with design thinking. Design Mgmt, 2015, Vol. 26(1), 18-23.

[17] W.F. Pruitt, Some Assembly Required: Structured problem solving demands structured communication, Quality Progress, 2020, Vol. 53(5), pp. 16-21.

[18] M. Altman et. Al, Design Thinking in Health Care. Preventing Chronic Disease, 2018, 180128.

[19] B. Ku and E. Lupton, Health Design Thinking. MIT Press, Cambridge, 2020.

[20] E.A. Neugebauer, A. Rath, ... and C. Gluud, Specific barriers to the conduct of randomised clinical trials on medical devices. Trials, 2017, Vol. 18(1), pp. 1-10.

[21] National Center for Advancing Translational Sciences. https://ncats.nih.gov/ accessed 6/2/2021

[22] Webpage of Trial Innovation Network. https://ncats.nih.gov/ctsa/projects/network accessed on 6/2/2021.

[23] A.M. Madni, Transdisciplinary Systems Engineering: Exploiting Disciplinary Convergence to Address Grand Challenges, IEEE Systems, Man, and Cybernetics Magazine, 2019, Vol. 5, No. 2, pp. 6-11.

[24] N.J. McNeese, The Pursuit of Transdisciplinary Research: Eight Recommendations for Integrating Disciplines. IEEE Systems, Man, and Cybernetics Magazine, 2019, Vol. 5(4), pp. 10-15. 